\title{
Transient laboratory X-ray absorption fine structure spectroscopy on thin films demonstrated with F8BT
}

Adrian Jonas, Katharina Witte, Birgit Kanngießer, Holger Stiel, loanna Mantouvalou

Adrian Jonas, Katharina Witte, Birgit Kanngießer, Holger Stiel, loanna Mantouvalou, "Transient laboratory X-ray absorption fine structure spectroscopy on thin films demonstrated with F8BT," Proc. SPIE 11886, International Conference on X-Ray Lasers 2020, 1188611 (8 July 2021); doi: $10.1117 / 12.2593245$ 


\title{
Transient laboratory $X$-ray absorption fine structure spectroscopy on thin films demonstrated with F8BT
}

\author{
Adrian Jonas $^{\mathrm{a}, \mathrm{e}}$, Katharina Witte ${ }^{\mathrm{b}}$, Birgit Kanngießer ${ }^{\mathrm{a}, \mathrm{c}, \mathrm{e}}$, Holger Stiel ${ }^{\mathrm{d}, \mathrm{e}}$ and Ioanna \\ Mantouvalou*a,c,e

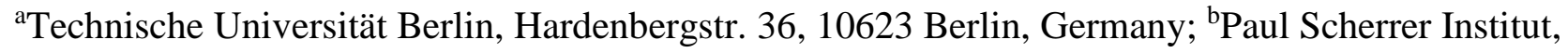 \\ Forschungsstr. 111, 5232 Villigen, Switzerland, current affiliation: Berlin Partner für Wirtschaft und \\ Technologie GmbH, Berlin, Germany ${ }^{c}$ Helmholtz Zentrum Berlin, Albert-Einstein-Str. 15, 12489 \\ Berlin, Germany; ${ }^{\mathrm{d}}$ Max-Born Institut Berlin, Max-Born-Strasse 2a, 12489 Berlin Germany; ${ }^{\mathrm{BL}} \mathrm{BX}$, \\ Berlin Laboratory for innovative X-ray technologies, Hardenbergstr. 36, 10623 Berlin, Germany;
}

\begin{abstract}
We present advanced instrumentation for the investigation of thin organic films offered by a laboratory X-ray absorption fine structure (XAFS) spectrometer for the soft X-ray range. The transmission spectrometer is based on a laser-produced plasma source in combination with a twin-arm reflection zone plate spectrometer. The efficiency and stability of the spectrometer allow for single shot measurements within $500 \mathrm{ps}$ with a resolving power of $\mathrm{E} / \Delta \mathrm{E} \sim 900$ in a range between $200 \mathrm{eV}$ and $1300 \mathrm{eV}$. Through the implementation of an optical pump beam, also transient absorption measurements can be performed. The merits of the spectrometer are demonstrated through the investigation of poly[(9,9-dioctylfluorenyl2,7-diyl)-alt-co-(1,4-benzo- $\left\{2,1^{‘}, 3\right\}$-thiadiazole)] (F8BT), a poly-fluorene copolymer. Transient optical pump soft X-ray probe spectroscopy with $500 \mathrm{ps}$ time resolution detects changes in the $\mathrm{C} \mathrm{K}$ edge spectrum which can be attributed to the lowest unoccupied molecular orbitals of the molecules in the benzothiadiazole unit.
\end{abstract}

Keywords: laboratory XAFS, soft X-rays, pump-probe spectroscopy, transient NEXAFS, F8BT

\section{INTRODUCTION}

Near edge X-ray absorption fine structure (NEXAFS) in the soft X-ray region as a means for the elucidation of the dynamics of electronic properties is still widely performed at large scale facilities. The ongoing development of brilliant laboratory soft X-ray sources enables an increasing number of transient NEXAFS experiments. These studies are widely focused on the investigation of ultrafast processes in the gas or liquid phase. But many scientific questions deal with dynamics in the solid phase, especially the field of organic electronics or photovoltaics can profit from new experimental possibilities for the investigation of thin organic films.

As an example the investigation of thin films of F8BT, an electronically conductive conjugated polymer, is selected. F8BT is used in a variety of organic electronics such as light emitting diodes due to its strong emission characteristics in the visible green light region [1] Here, not only the electronic properties of the molecule as such are of interest, but the changes in properties dependent on the manufacturing of the organic film such as the orientation of the molecules.

We present a laboratory soft X-ray NEXAFS spectrometer which is optimized for the investigation of thin organic films. It operates in the soft X-ray regime up to about $1300 \mathrm{eV}$, where not only the main constituents of organic molecules can be probed, but also the L edges of transition metals, which often play a central role in the properties of such molecules. After presenting the sample, its preparation and the experimental setup, the static and transient $\mathrm{C} K$ edge NEXAFS spectra of F8BT are shown and discussed.

* Ioanna.mantouvalou@helmholtz-berlin.de

International Conference on X-Ray Lasers 2020, edited by Davide Bleiner,

Proc. of SPIE Vol. 11886, 1188611 - (c) 2021 SPIE · CCC code:

$0277-786 \mathrm{X} / 21 / \$ 21 \cdot$ doi: $10.1117 / 12.2593245$

Proc. of SPIE Vol. $118861188611-1$ 


\section{MATERIALS AND METHODS}

\subsection{Materials}

The organic molecule used in our investigations is called F8BT, short for poly[(9,9-dioctylfluorenyl-2,7-diyl)-alt-co(1,4-benzo- $\left\{2,1^{`}, 3\right\}$-thiadiazole)]. It is a poly-fluorene copolymer with deep lying highest unoccupied molecular orbital (HOMO), lowest unoccupied MO (LUMO) levels $(\mathrm{HOMO}=-5.9 \mathrm{eV}, \mathrm{LUMO}=-3.3 \mathrm{eV}$ [2]), see figure 1, where the HOMO level is delocalized over the entire carbon backbone, and the LUMO orbitals are in the benzothiadiazole (BT) group. The calculation of the HOMO and LUMO wavefunctions was performed using ORCA 4.1 with B3LYP/def2TZVP level of theory and the isosurfaces were rendered using Avogadro 1.2. Upon electric or light excitation the molecule is excited into the S1 or higher singlet state, then relaxes through S1 into a long-lived triplet state. Delayed fluorescence from the spin allowed singlet transitions is observed due to triplet migration with successive triplet-triplet annihilation or thermally activated delayed fluorescence [3].
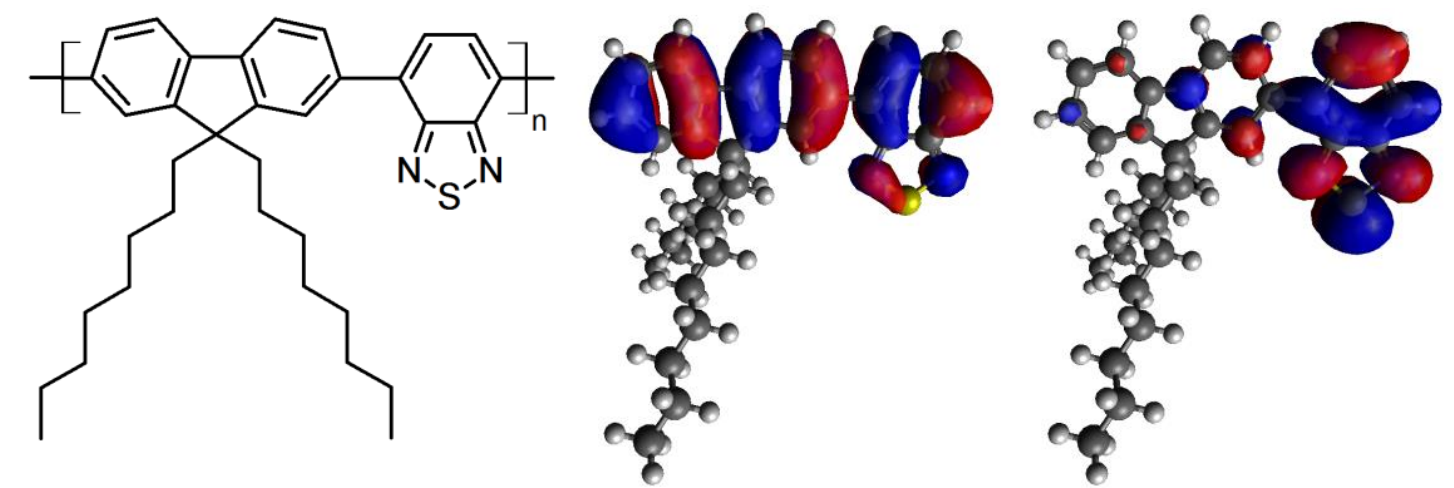

Figure 1: The molecule F8BT is composed of a carbon backbone with aromatic structures and alkyl side chains (left). The wavefunction isosurfaces of the HOMO (middle) and LUMO (right) show the localization of the LUMO in the BT unit.

The organic thin films were prepared via blade coating on microscopy glass slides at room temperature, see figure 2 . For this purpose the glass slides were first coated with a thin film $(<20 \mathrm{~nm})$ of poly $(3,4$-ethylendioxythiophene $)$ polystyrene sulfonate (PDOT:PSS) to better remove the F8BT film afterwards. The F8BT fibers with a mean molecular weight of 82 $\mathrm{kDa}$ and $111 \mathrm{kDa}$ were dissolved in p-xylene (anhydrous, 99\%, Sigma Aldrich) with a final concentration of $30 \mathrm{mg} / \mathrm{ml}$ in the polymer solution. After blade coating the samples were cut into small pieces $(<1 \mathrm{~mm} \times 1 \mathrm{~mm})$ and floated of the glass substrate in water. Successively, the film samples were picked up with $\mathrm{Si}_{3} \mathrm{~N}_{4}$ membranes (Silson Ltd, $100 \mathrm{~nm}$ thick, $1 \mathrm{~mm} \times 1 \mathrm{~mm}$ window size) and annealed for $30 \mathrm{~min}$ at $290^{\circ} \mathrm{C}$, a temperature above the melting temperature of F8BT, see figure 2, right. As a last step the samples were quenched to room temperature on a copper plate. The resulting films were analyzed with AFM resulting in a thickness estimation of $150 \mathrm{~nm}+/-50 \mathrm{~nm}$.

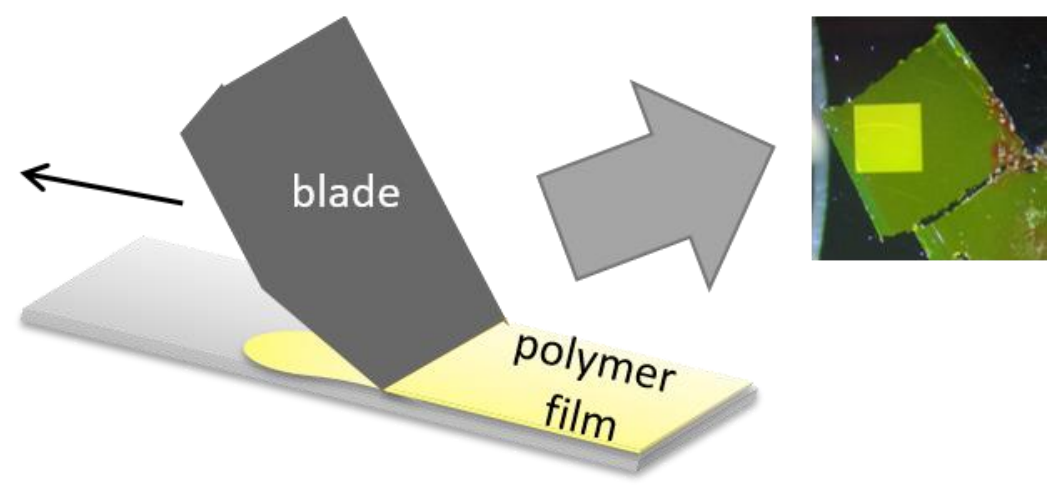

Figure 2: A schematic of the used blade coating technique and a photograph of the thin film on a $\mathrm{Si}_{3} \mathrm{~N}_{4}$ window frame. 


\subsection{Methods}

The prepared films were first analyzed with absorption spectroscopy using UV/Vis light using a Lambda 900 spectrometer by Perkin Elmer. Sample and reference spectra are collected simultaneously with $0.5 \mathrm{~nm}$ step width and $100 \mathrm{~nm} / \mathrm{min}$ measurement velocity. Three measurements were performed and the mean spectra are displayed in figure 3. The spectra show three absorption bands in line with literature $[4,5]$ which can be attributed to the transitions into excited singlet states. The third harmonic of the driving laser for the plasma source at $343 \mathrm{~nm}$ is, thus, well suited for excitation into the $S_{2}$ state which will relax ultrafast into $S_{1}$ and through intersystem crossing to the first triplet state $T_{1}$. For the following NEXAFS measurements only the film with $82 \mathrm{kDa}$ was analyzed, as both films show similar optical characteristics.

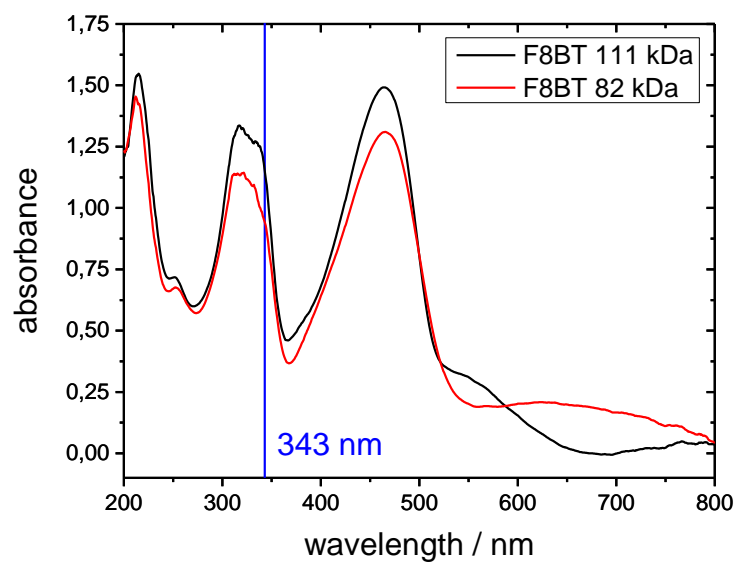

Figure 3: UV/Vis spectrum of the prepared films.

The used laser-produced plasma source [6] emits polychromatic radiation with a pulse length of $500 \mathrm{ps}$ at $100 \mathrm{~Hz}$. The broadband isotropic radiation is transmitted through the sample and a reference and dispersed via a pair of identical reflection zone plate structures onto a CCD camera, see figure 4. Different stabilization mechanisms using pinhole cameras ensure that the collected spectra have a low uncertainty following Poisson statistics. For the study of transient spectra, a pump beamline using a small portion of the driving laser is available with tunable wavelength and time delay. A detailed description of the instrument can be found in previous work [7].

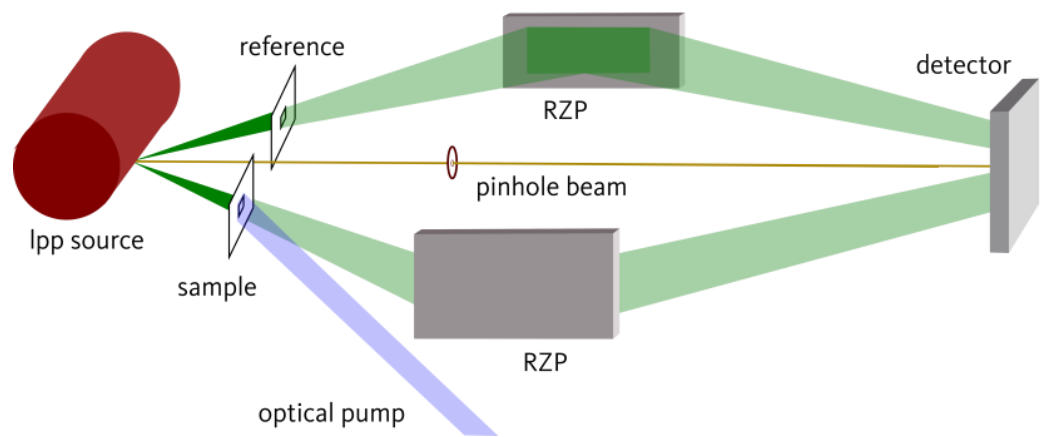

Figure 4: Schematic view of the presented spectrometer.

For the presented transient NEXAFS measurements, the pump beam wavelength was set to $343 \mathrm{~nm}$ by using two nonlinear crystals (BBO). The diameter of the visible light beam was expanded to $3.3 \mathrm{~mm}$ FWHM on the sample window at 
$1.5 \mathrm{~mJ}$ per pulse, resulting in a photon flux density of $10^{26} \mathrm{ph} / \mathrm{s} / \mathrm{cm}^{2}$. The laser energy used for generation of the probe Xray beam was set to $110 \mathrm{~mJ}$. The measurements were performed in single shot mode with 8 -fold hardware binning of the CCD camera, so that 6000 single shot images result in a measurement time of $4 \mathrm{~h}$. To minimize source instabilities the spectra were collected with alternating laser pump on and off, resulting in 3000 static and 3000 pumped spectra. 50 background images were collected before and after every 2000 images. The energy axis was obtained by measuring a copper target emission spectrum and calibrating it using tabulated data for highly ionized atomic copper lines [8]. This results in an absolute energy calibration of $0.2 \mathrm{eV}$ [9]. Due to pinhole camera stabilization mechanisms, the energy axis remains constant for all further measurements.

\section{RESULTS}

Figure 5 shows the resulting static NEXAFS spectra at the $\mathrm{C} K$ edge as a function of number of accumulated single shots. Using isolated copper emission lines the resolving power $\mathrm{E} / \Delta \mathrm{E}$ was determined to be 950 . The spectra are dominated by three structures, which will be described later in detail. All three features are already discernible in a single shot spectrum, see figure 5 .

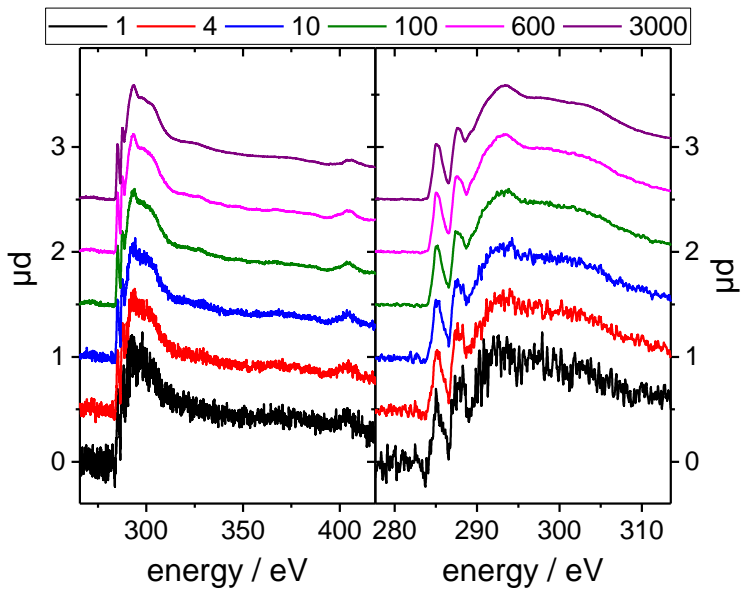

Figure 5: C K edge NEXAFS spectra as a function of the number of accumulated images. Already in a single shot spectrum all relevant features are discernible

Table 1: Standard deviation as a function of the number of accumulated spectra in two different energy regimes.

\begin{tabular}{|l|l|l|}
\hline $\begin{array}{l}\text { Number } \begin{array}{l}\text { accumulated } \\
\text { images }\end{array} \\
\text { of }\end{array}$ & $\begin{array}{l}\text { Standard } \\
\text { deviation in the } \\
\text { energy range } \\
253,956 \mathrm{eV} \text { to } \\
283,76 \mathrm{eV}\end{array}$ & $\begin{array}{l}\text { Standard } \\
\text { deviation in the } \\
\text { energy range } \\
342,86 \mathrm{eV} \text { to } \\
380,25 \mathrm{eV}\end{array}$ \\
\hline 1 & 0,06 & 0,04 \\
\hline 10 & 0,03 & 0,02 \\
\hline 100 & 0,02 & 0,014 \\
\hline 600 & 0,010 & 0,007 \\
\hline 3000 & 0,008 & 0,006 \\
\hline
\end{tabular}

In table 1 the calculated standard deviations in two energy ranges, one before and the other after the absorption edge are listed. The differences here are caused by the different number of photons in the two regions, depending on two parameters: the absorption of the sample, but also the emitted photons from the source. In the presented case, the latter 
effect is dominant. As can be seen, changes in absorption in the range of $10^{-3}$ can be detected already with a few hundred of single shots rendering transient measurements with high accuracy feasible. While this takes about 24 min when using a CCD camera as detector, measurement times can be reduced drastically when using a sCMOS camera [10] to a few seconds.

Figure 6 shows the static $\mathrm{C}$ K edge NEXAFS spectrum in detail. The vertical numbered lines are reference values taken from [11] which were shifted by $0.11 \mathrm{eV}$ for a better match to the experimental spectrum. (Note as stated above that the described absolute energy calibration has an uncertainty of $0.2 \mathrm{eV}$.) The publication lists 6 features, mostly in the first peak.

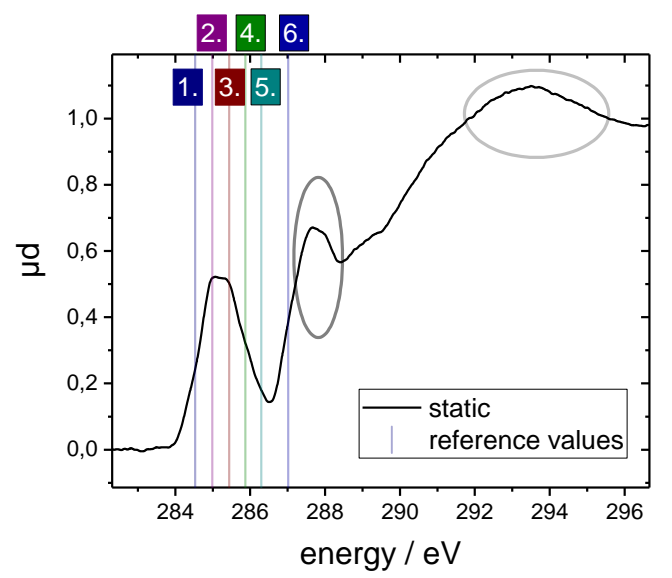

Figure 6: The first three peaks of the NEXAFS spectrum with reference values [11]

The non-resolved split peak at $285 \mathrm{eV}$ originates from fluorenyl $\mathrm{C} 1 \mathrm{~s}(\mathrm{C}=\mathrm{C})->$ pi* as for example seen in polydioctylfluorene (PFO). In contrast to PFO the peak is split into five features due to interactions of the fluorenyl and BT structures [11]. Also the sixth feature, which is apparent as a shoulder of the second peak can be attributed to this interaction for the same reasons. The main component of the second peak around $287.5 \mathrm{eV}$ (dark grey circle in figure 6) is attributed to $1 \mathrm{~s}(\mathrm{C}-\mathrm{H})$-> pi* from the saturated dioctyloxy side chains. This assumption is backed by the observation that this feature is not significantly sensitive to the orientation of the aromatic structures in the film. Finally, broader resonances at $293 \mathrm{eV}$ (light grey circle in figure 5) and $300 \mathrm{eV}$ (not shown in figure 6) derive from 1s (C-C) -> sigma* transitions [12].
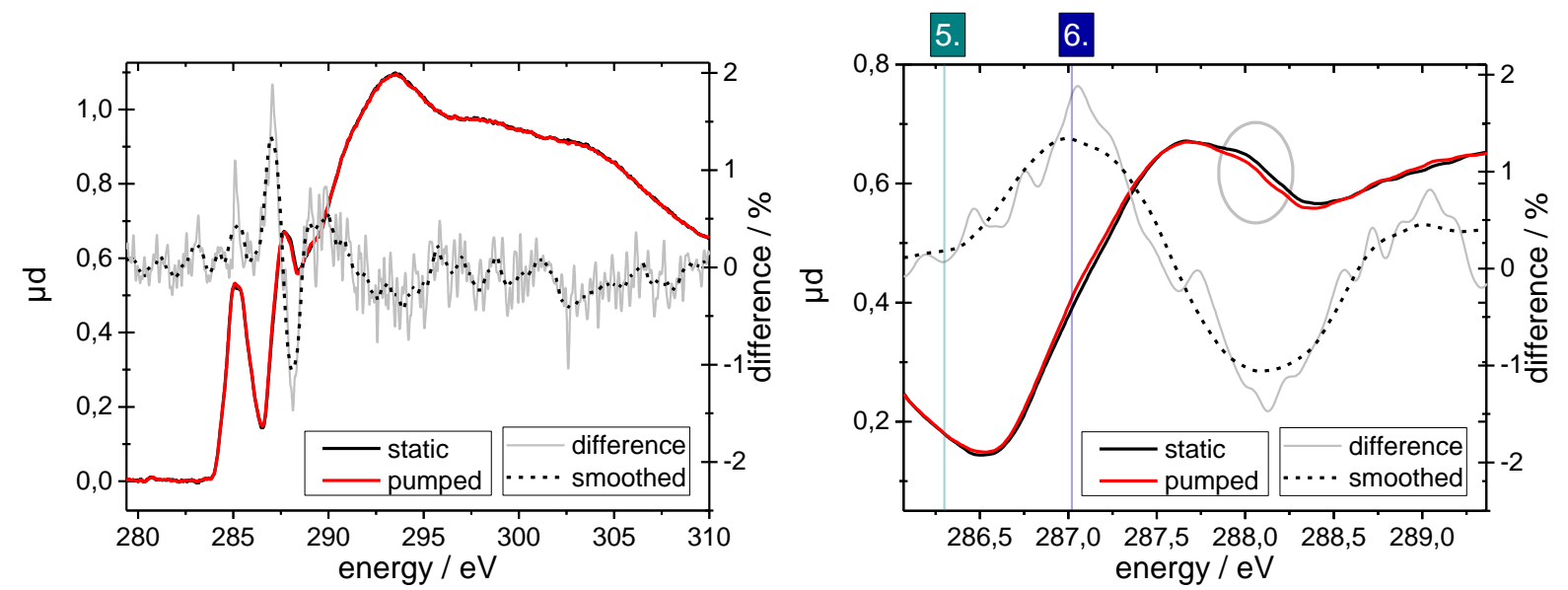

Figure 7: Transient C Kedge spectrum: left: full spectrum, right: detail of the second peak.

Figure 7 presents the transient measurement with the static NEXAFS spectrum from figure 6 in black, the pumped NEXAFS spectrum in red and the transient absorption spectrum as a grey line. The black dashed line is a smoothed representation for clarity purposes. A significant change is only visible around the structure at $287.5 \mathrm{eV}$. The sixth 
feature linked to the BT group shows an increase, while a different feature which has not been attributed yet decreases (grey circle in figure 7, right).

So, what is visible in nanosecond time scales, is, that there is a change in the electronic structure linked to the BT group, which is exactly the location of the LUMO orbital. This is well in line with the understanding of the processes concerning F8BT and for example fluorescence decay measurements which propose a time constant of about $900 \mathrm{ps}$ [13].

\section{DISCUSSION AND CONCLUSIONS}

A laboratory setup for optical-pump soft X-ray-probe NEXAFS spectroscopy is presented which is adapted to the transmission measurement of thin films. The possibility to perform transient spectroscopy is offered through an optical pump beamline (see also Sects. 1-4 [14]).

The sample is irradiated on a large area, minimizing radiation damage. Additionally, as sample and reference spectra are collected in single shot mode, with each shot of the source, the full NEXAFS spectrum is collected. That means, that possible radiation damage, originating either through the soft X-rays or the visible laser light, can be monitored using the full spectral information. This is in contrast to typical synchrotron radiation setups, where, when measuring the full spectra, the energy must be scanned, so that radiation damage results in distortion of the spectra, rendering it difficult to determine the exact spectral change due to the damage. Pumped and un-pumped spectra are collected in an alternating way, additionally facilitating the differentiation of laser and X-ray effects.

With the setup single shot spectroscopy is feasible, demonstrated with the example of a thin film of F8BT, which is a well-studied molecule used for organic electronics. To the best of our knowledge we present the first transient optical pump soft X-ray probe spectra of such a film. The changes in the absorption spectrum at the $\mathrm{C}$ K edge are located at the BT group of the molecule, which is the location of the LUMO. As we probe the changes with 500 ps pulses, nanosecond time constants are expected, which is well in line with optical fluorescence measurements [13]. The reason for this long lived dynamics can be explained by delayed fluorescence in the molecule due to triplet migration over the extended C backbone. As an outlook, time resolved measurements as well as DFT calculations of the C K edge NEXAFS spectrum can be exploited to further understand the nanosecond dynamics.

\section{REFERENCES}

[1] Xia, R. D., Campoy-Quiles, M., Heliotis, G., Stavrinou, P., Whitehead, K. S., Bradley, D. D. C., "Significant improvements in the optical gain properties of oriented liquid crystalline conjugated polymer films", Synthetic Metals 155, 274-278 (2005).

[2] Zhang, Y. et al., "Electron and hole transport in poly(fluorene-benzothiadiazole)", Appl. phys. Lett., Vol 98, 143504 (2011).

[3] Dey A., Chandrasekaran N., Chakraborty D., Johari P., McNeill C.R., Rao A., Kabra D., "Kinetics of thermally activated triplet fusion as a function of polymer chain packing in boosting the efficiency of organic light emitting diodes", npj Flex Electron 2, 28 (2018)

[4] Liu C., Xu Y., Liu X., Minari T., Sirringhaus H., Noh Y.-Y., "Solution-processed high-LUMO-level polymers in n-type organic field-effect transistors: a comparative study as a semiconducting layer, dielectric layer, or charge injection layer" Semicond. Sci. Technol. 30, 044007 (2015).

[5] Hashim Z., Alomari S., Alghamdi W., Altuwirqia R., Green M., "Optically observed multiple inter-chain interactions in polyblend semiconducting polymer nanoparticles", RSC Adv., 7, 48308-48314 (2017)

[6] Mantouvalou I., Witte K., Grötzsch D., Neitzel M., Günther S., Baumann J., Jung R., Stiel H., Kanngießer B., Sandner W., "High average power, highly brilliant laser-produced plasma source for soft X-ray spectroscopy", Review of Scientific Instruments 86, 035116 (2015).

[7] Jonas A., Stiel H., Glöggler L., Dahm D., Dammer K., Kanngießer B., Mantouvalou I., "Towards Poisson noise limited optical pump soft X-ray probe NEXAFS spectroscopy using a laser-produced plasma source", Optics Express 27:25, 36524-36537 (2019)

[8] Shirai, T., "Spectral Data and Grotrian Diagrams for Highly Ionized Copper, $\mathrm{Cu} \mathrm{X}$-Cu XXIX", Journal of Physical and Chemical Reference Data 20, 1 (1991). 
[9] Mantouvalou, I., "Single shot near edge x-ray absorption fine structure spectroscopy in the laboratory," Appl. Phys. Lett. 108, 201106 (2016).

[10] Jonas A., Staeck S., Kanngießer B., Stiel H. Mantouvalou I., "Laboratory quick near edge X-ray absorption fine structure spectroscopy in the soft X-ray range with $100 \mathrm{~Hz}$ frame rate using CMOS technology“, Rev. Sci. Instrum., review process

[11] Watts, B., Swaraj S., Nordlund D., Lüning J., Ade H., "Calibrated NEXAFS spectra of common conjugated polymers", J. Chem. Phys. 134, 024702 (2011).

[12] Watts B., Schuettfort T., McNeill C.R., "Mapping of Domain Orientation and Molecular Order in Polycrystalline Semiconducting Polymer Films with Soft X-Ray Microscopy”, Adv. Funct. Mater. 21, 1122-1131 (2011)

[13] Devižis A., Serbenta A., Meerholz K., Hertel D., Gulbinas V., " Excited state relaxation in poly(spirobifluorene-co-benzothiadiazole) films“, J. Chem. Phys. 131, 104902 (2009)

[14]D. Bleiner, The Science and Technology of X-ray Lasers: A 2020 Update Proc. SPIE 11886, 1188602 (2021) 